Vahid Eslami, MD

Neurology Researcher

1- Sina Trauma and Surgery Research Center,

Sina Hospital
Tehran University of Medical Sciences

Tehran, Iran

E-mail:v_rahimi@tums.ac.ir

\title{
References
}

1. Magalhaes FN, Dotta L, Sasse A, Teixera MJ, Fonoff ET. Ozone therapy as a treatment for low back pain secondary to herniated disc: A systematic review and meta-analysis of randomized controlled trials. Pain Physician 2012:15: E115-129.

2. Sagai M, Bocci V. Mechanisms of action involved in ozone therapy: Is healing induced via a mild oxidative stress? Med Gas Res 2011; 1:29.

3. Borrelli E. Mechanism of action of oxygen ozone therapy in the treatment of disc herniation and low back pain. Acta Neurochir Suppl 2011; 108:123-125.

4. Muto M, Ambrosanio G, Guarnieri G, Capobianco E, Piccolo G, Annunziata G, Rotondo A. Low back pain and sciatica: Treatment with intradiscal-intraforami- 6 . nal O(2)-O (3) injection. Our experience. Radiol Med 2008; 113:695-706.

5. Paoloni M, Di Sante L, Cacchio A, Apuzzo D, Marotta S, Razzano M, Franzini M, Santilli V. Intramuscular oxygen-ozone therapy in the treatment of acute back pain with lumbar disc herniation: A multicenter, randomized, double-blind, clinical trial of active and simulated lumbar paravertebral injection. Spine (Phila $\mathrm{Pa}$ 1976) 2009; 34:1337-1344.

6. Bonetti M, Fontana A, Cotticelli B, Volta GD, Guindani M, Leonardi M. Intraforaminal $\mathrm{O}(2)-\mathrm{O}(3)$ versus periradicular steroidal infiltrations in lower back pain: Randomized controlled study. AJNR Am J Neuroradiol 2005; 26:996-1000.

\section{Obtaining More Information from the Sacroiliac Joint Arthrogram}

\section{TO THE EDITOR:}

We applaud Dr. Hansen and colleagues on their systematic evaluation of the therapeutic effectiveness of sacroiliac joint injections (1). The review provided insight into our treatment of a capacious joint, the sacroiliac (SI) joint, which represents a small percentage, in terms of incidence, of all low back pain cases. However, when a degenerative SI joint is the etiology of symptoms, this may result in severe symptoms and may significantly affect lumbosacral spine pathology and pain. Despite the complex anatomy of the SI joint, many of our colleagues deserve praise for their attempts to optimize treatment of it using diagnostic interventional techniques to ensure better therapeutic outcomes, such as the double needle technique (2). It is intuitive that a more definitive diagnosis would ensure a better therapeutic outcome regardless of the therapeutic modality chosen by the treating physician. Dr. Hansen's review has shown that this is not the case, except in the case of using cooled radiofrequency neurotomy, which demonstrated a "fair" therapeutic benefit. In today's health care climate, superior and enhanced results are demanded from us by our patients. Less robust or unsuccessful results also invite our opponents (including the insurance industry) to further launch attacks against our specialty wherein they claim that our ap- proaches lack evidence and are "experimental."

After reading the present review (1), and after performing a recent sacroiliac joint injection with surprising results, it dawned on us that the problem may not be inherent to the technical aspects of the therapeutic SI joint procedure itself, but rather to the possibility that we are choosing the wrong interventional procedure for the right diagnosis. In other words, depending on the incompetency of the sacroiliac joint, therapeutic results probably vary. During a recent $\mathrm{SI}$ joint injection, we decided to inject a larger volume of water-soluble, iodine-based contrast medium $(4 \mathrm{~mL})$ than that which is typically utilized for diagnostic purposes (less than one $\mathrm{mL}$ ). Surprisingly, the contrast medium travelled throughout the entire joint, and exited at the superior region revealing a "water geyser" sign (Figs. 1, 2, 3). After further studying the films, it was obvious that the joint was too incompetent to retain any injectate, and any interventional pain treatment procedure would likely fail to provide long-term relief. Such a patient would probably benefit from a more advanced surgical treatment, such as a lumbar spine fusion. Interpreting the arthrogram more in-depth can allow for a better therapeutic outcome for the patient, and prevent unnecessary procedures, which ultimately leads to overall 

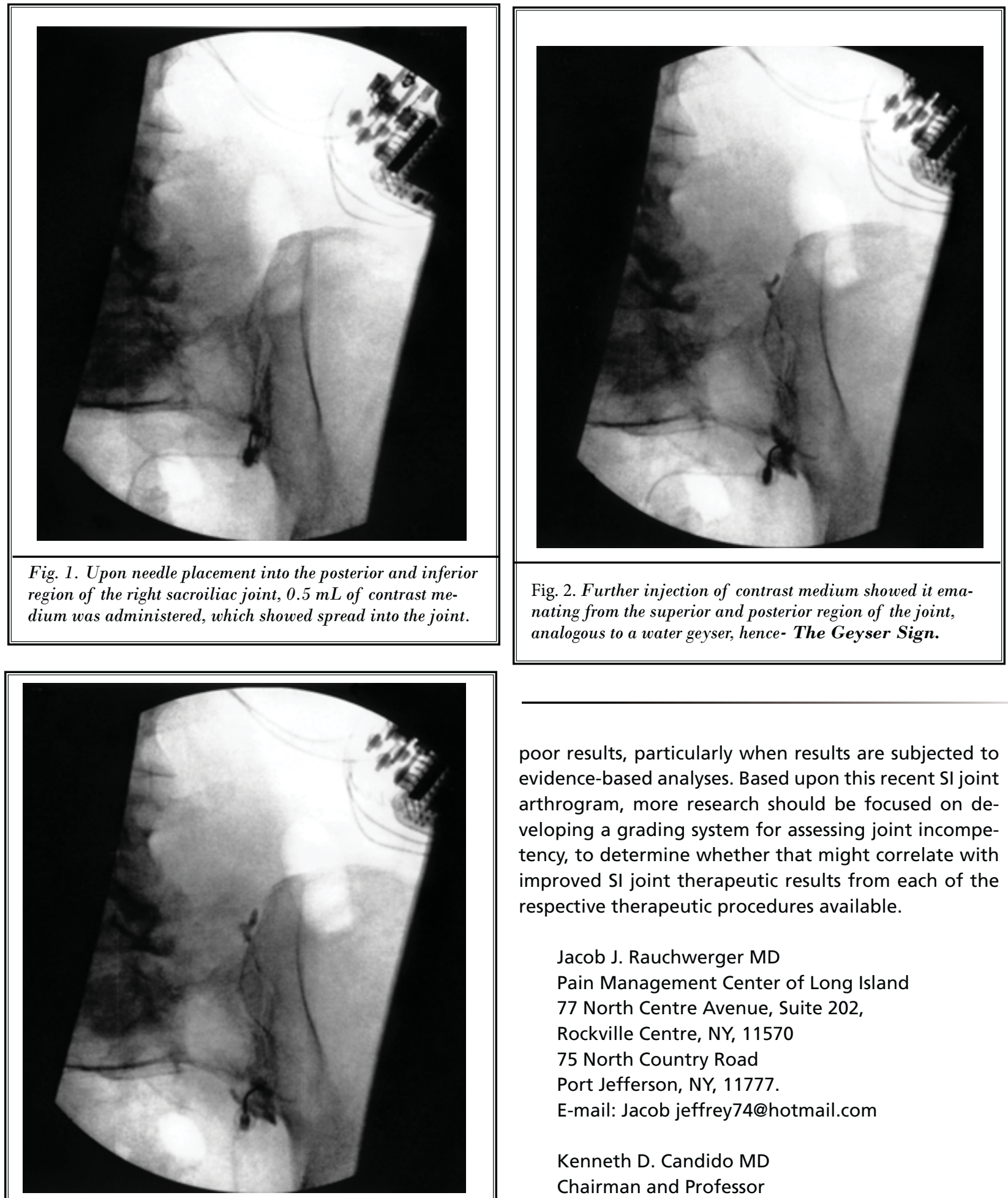

Fig. 3. Further contrast medium injection increased the "Geyser Sign" height, however, there would also be backflow from the posterior and inferior region of the joint.

poor results, particularly when results are subjected to evidence-based analyses. Based upon this recent $\mathrm{SI}$ joint arthrogram, more research should be focused on developing a grading system for assessing joint incompetency, to determine whether that might correlate with improved SI joint therapeutic results from each of the respective therapeutic procedures available.

\section{Jacob J. Rauchwerger MD}

Pain Management Center of Long Island 77 North Centre Avenue, Suite 202,

Rockville Centre, NY, 11570

75 North Country Road

Port Jefferson, NY, 11777.

E-mail: Jacob jeffrey74@hotmail.com

Kenneth D. Candido MD

Chairman and Professor

Department of Anesthesiology

Advocate Illinois Masonic Medical Center

836 W. Wellington Ave, Suite 4815

Chicago, IL, 60657 


\section{References:}

1. Hansen H, Manchikanti L, Simopoulous T, Christo PJ, Gupta S, Smith HS, Hammed H, Cohen SP. A systematic evaluation of the therapeutic effec- tiveness of sacroiliac joint interventions. Pain Physician 2012; 15:E247-E278.

2. Gupta S. Double needle technique: An alternative method for performing diffi- cult sacroiliac joint injections. Pain Physician 2011; 14:281-284.

\section{In response}

Thank you Drs. Rauchwerger and Candido, for your valued critique and contribution.

The sacral joint is an elusive pain generator that has elicited much controversy in the past century. Prior to Mixter and Barr alleging discogenic pathology as a painful source of low back pain in 1931, the sacral joint was felt to be the primary source of most low back pain. Physicians have been mutilating the disc in various ways ever since.

Reenter the sacral joint. The passage of time has resulted in inevitable human lifestyle changes, our physical habitus grows, and we find new ways to advance self destructive approaches to promote spine pain (cigarettes, wrecks, etc.). The sacral joint is again finding an expanded role in the differential diagnosis of back pain. Acknowledging that bedside testing is not as robust as we would like, and with the exception of specific disease entities such as ankylosing spondylitis, imaging is not always rewarding. To promote evidenceand outcome-based practice, the most potent diagnostic tool to define sacral joint pain is a fluoroscopically guided local anesthetic block. Once we have diagnosed the sacral joint as a pain generator, we often wonder what to do when the pain returns. It makes sense to consider advanced techniques such as ablation, based on a patient's documented decline in quality of life indices. When asked by the payers where the outcome evidence clarifies the decision making, we sometimes struggle. Diagnosing spinal pain is as difficult a problem as any diagnosis in medicine.

A 1995 editorial in the British Medical Journal reminded us that only $15 \%$ of medical interventions are supported by solid scientific evidence. Furthermore, a scant $21 \%$ of 126 diagnostic and therapeutic technologies assessed by the National Institutes of Health were firmly based in generated scientific evidence. Dr. Shultz, and others, give us 3 points to ponder:

1. The absence of randomized controlled data to support an intervention is not the same as evidence against it.
2. Data that are not randomized and controlled are not necessarily worthless.

3. When a treatment has withstood the test of time, and has a theoretical basis and literature to support it, we should not be quick to abandon it.

Dr. Rauchwerger is correct: this is a complex joint. On a daily basis, insurance companies and third party payers scrutinize the multiple techniques we use in interventional pain management. Procedures are denied, deemed "not medically necessary" or "experimental" despite evidence of their diagnostic and therapeutic benefit. More likely, denial of therapeutic effectiveness is tied to cost and overutilization. The treatment of sacral joint pain has stood the test of time, is a diagnostic and therapeutic challenge, and deserves advanced therapies.

Drs. Rauchwerger and Candido also appreciate that a plausible explanation for interventional failure exists, despite excellent technique. Maybe it is time for some type of grading system to better understand responses to therapy, which would lend consistency to documentation of medical necessity and outcome assessment. Multiple societies coming forward with this type of tool is only in the best interest of the patient, as are the ongoing advancement of pain therapies, both minimally invasive and surgical. Certainly the mere fact that surgical options are available, given the authority of the diagnostic technique, speaks for the value of any intervention. This is a truth Dr. Rauchwerger correctly elaborates. We continue to learn much from the sacral joint.

\author{
Hans C. Hansen, MD \\ Medical Director \\ The Pain Relief Centers, LLC \\ P.O. Box 939 \\ Conover, NC 28613 \\ E-mail: hhansen@painreliefcenters.com
}

